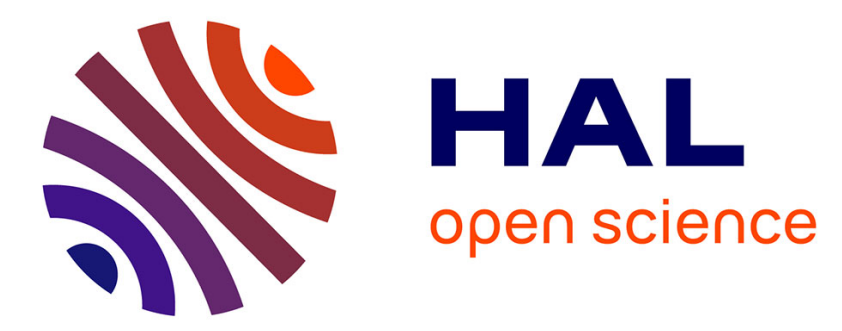

\title{
Thermal study of an aluminium nitride ceramic heater for spray CVD on glass substrates by quantitative thermography
}

Olivier Riou, Pierre-Olivier Logerais, Vincent Froger, Jean-Félix Durastanti, Anne Bouteville

\section{To cite this version:}

Olivier Riou, Pierre-Olivier Logerais, Vincent Froger, Jean-Félix Durastanti, Anne Bouteville. Thermal study of an aluminium nitride ceramic heater for spray CVD on glass substrates by quantitative thermography. Quantitative InfraRed Thermography Journal, 2013, 10 (2), pp.159-171. 10.1080/17686733.2013.793468 . hal-01207446

\section{HAL Id: hal-01207446 \\ https://hal.science/hal-01207446}

Submitted on 30 Sep 2015

HAL is a multi-disciplinary open access archive for the deposit and dissemination of scientific research documents, whether they are published or not. The documents may come from teaching and research institutions in France or abroad, or from public or private research centers.
L'archive ouverte pluridisciplinaire HAL, est destinée au dépôt et à la diffusion de documents scientifiques de niveau recherche, publiés ou non, émanant des établissements d'enseignement et de recherche français ou étrangers, des laboratoires publics ou privés. 


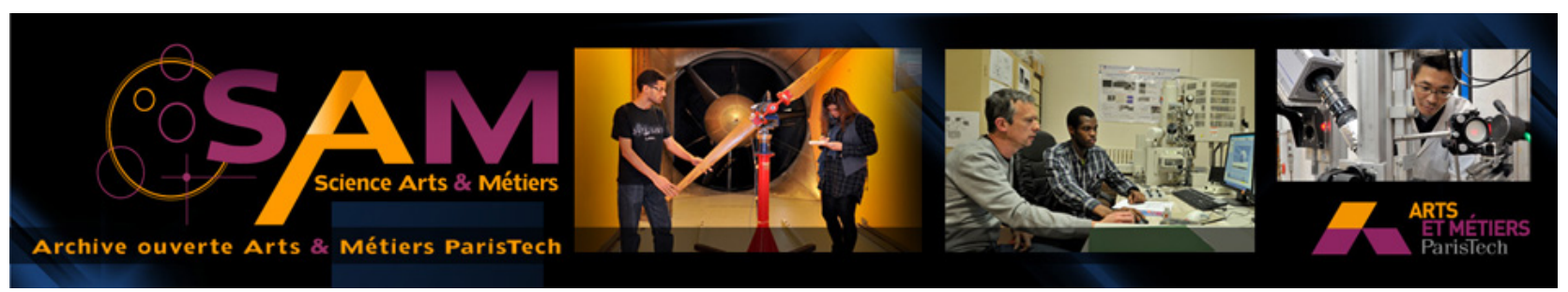

\section{Science Arts \& Métiers (SAM)}

is an open access repository that collects the work of Arts et Métiers ParisTech researchers and makes it freely available over the web where possible.

This is an author-deposited version published in: http://sam.ensam.eu

Handle ID: .http://hdl.handle.net/10985/10286

\section{To cite this version :}

Olivier RIOU, Pierre-Olivier LOGERAIS, Vincent FROGER, Jean-Félix DURASTANTI, Anne BOUTEVILLE - Thermal study of an aluminium nitride ceramic heater for spray CVD on glass substrates by quantitative thermography - Quantitative InfraRed Thermography Journal - Vol. 10, $n^{\circ} 2$, p. $159-171-2013$ 


\title{
Thermal study of an aluminium nitride ceramic heater for spray CVD on glass substrates by quantitative thermography
}

\author{
Olivier Riou ${ }^{\mathrm{a}}$, Pierre-Olivier Logerais ${ }^{\mathrm{a} *}$, Vincent Froger ${ }^{\mathrm{b}}$, Jean-Félix Durastanti $^{\mathrm{a}}$ and Anne \\ Bouteville $^{\mathrm{b}}$ \\ ${ }^{a}$ CERTES, IUT de Sénart, Université Paris-Est Créteil, rue Georges Charpak, Lieusaint, France; \\ ${ }^{b}$ LAMPA, Arts et Métiers ParisTech, Angers, France
}

(Received 19 December 2012; final version received 28 March 2013)

\begin{abstract}
A thermographic approach is used to determine the temperature of an aluminium nitride hot plate as a glass substrate heater for depositing thin films by spray Chemical Vapour Deposition. In this context, the temperature of the hot plate is conditioned by the evaluation of both effective emissivity and environment temperature with a calibration curve of the commercial camera. We first examined the consistency of the thermosignal/temperature correspondence by employing the software calibration. The environment temperature is evaluated by means of a ruffled aluminium foil according to ASTM. The effective emissivity is measured in situ by using a commercial IR camera in the temperature range 40$540{ }^{\circ} \mathrm{C}$ with a better than $3 \%$ accuracy. Absolute value of effective emissivity is in agreement with spectrometric values up to $120^{\circ} \mathrm{C}$. Above this temperature, a strong dependence with temperature is highlighted. The radiometric temperature values are, thereby, corrected with an exactitude of temperature better than $2.5 \%$ in Celsius degree for the highest temperatures considered.
\end{abstract}

Keywords: hot plate heating; effective emissivity; spectral emissivity; radiometric temperature correction; measurement uncertainty

\section{Introduction}

Spray Chemical Vapour Deposition (CVD) is a thin film deposition technique where fine liquid droplets are projected onto a heated glass substrate (Figure 1) [1]. With this method, coatings for solar cells such as transparent conducting oxides can be deposited in a cheap way $[2,3]$. However, one of the remaining challenges is to have a uniform substrate temperature in order to get homogeneous thin film deposition. Consequently, the heating of the glass substrate has to be mastered before depositing thin films. Different ways exist to heat up the glass substrate such as the use of halogen lamps or the utilisation of a hot plate [4]. In the present work, the heating provided by a hot plate is investigated prior to all deposition considerations, with the aim of having a better knowledge of its temperature values. For this purpose, among temperature measurement techniques, infrared thermography has been chosen as it is a convenient non-contact temperature measurement means to determine temperature mapping. After having exposed the thermographic method employed, the experimental set-up is

*Corresponding author. Email: pierre-olivier.logerais@u-pec.fr 


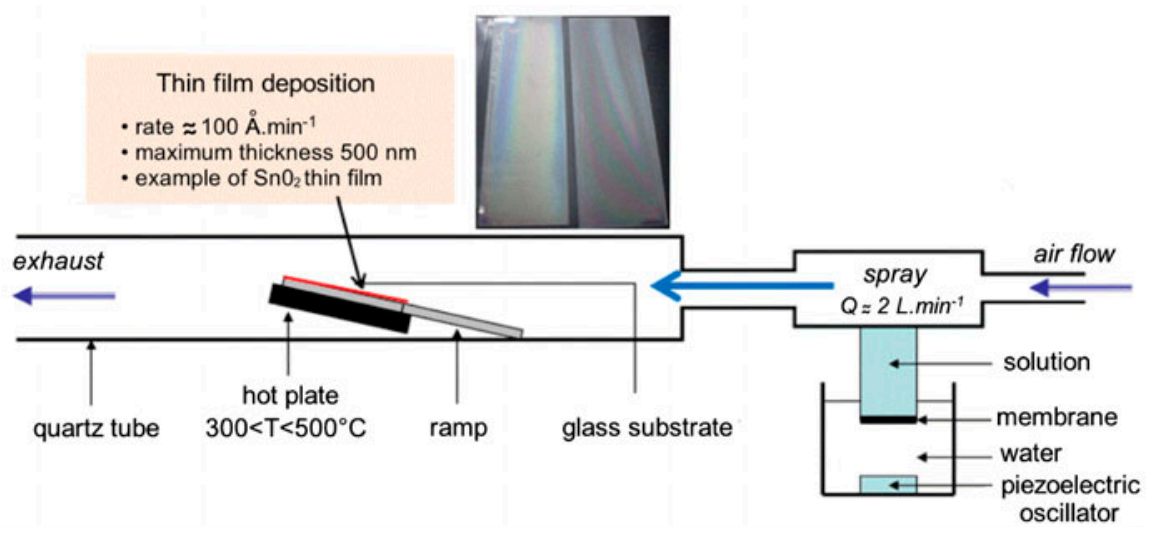

Figure 1. Spray CVD with hot plate heating.

presented. The different stages leading to the hot-plate temperature evaluation are then described and the obtained temperature results are discussed.

\section{Quantitative thermography}

The infrared thermography utilises the intensity of thermal radiation emitted by a target in order to infer the surface temperature. The intensity is measured over a spectral bandwidth $\Delta \lambda$, which is characteristic of any infrared systems. Excluding atmospheric contribution, the intensity of radiation of an opaque body can be modelled as:

$$
L_{\Delta \lambda}\left(T_{\text {app. }}\right)=L_{\Delta \lambda, e}(T)+L_{\Delta \lambda, \text { refl. }}^{0}\left(T_{\text {env. }}\right)
$$

where $L_{\Delta \lambda, e}(T)$ is the spectral radiance of the target at surface temperature $T, L_{\Delta \lambda, \text { refl. }}^{0}\left(T_{\text {env. }}\right)$ is the spectral radiance of the thermal environment (considered as a perfect emitter) at an equivalent temperature $T_{\text {env }}$ and reflected by the target surface. These intensities are referenced to the one of a perfect absorber and perfect emitter, a blackbody, whose spectral radiance is given by the Planck distribution:

$$
\begin{aligned}
& L_{\Delta \lambda, e}(T)=\int_{\Delta \lambda} \varepsilon(\lambda, T) \times L^{0}(\lambda, T) d \lambda \\
& L^{0}(\lambda, T)=\frac{c_{1}}{\lambda^{5}\left(e^{\frac{c_{2}}{\lambda T}}-1\right)}
\end{aligned}
$$

where $c_{1}=1.19 \times 10^{8} \mathrm{~W} \mu \mathrm{m}^{4} \mathrm{~m}^{-2} \mathrm{sr}^{-1}, c_{2}=1.439 \times 10^{4} \mu \mathrm{mK}$. These relations can be implemented indifferently for the target of spectral emissivity $\varepsilon(\lambda, T)$ and for the environment of spectral emissivity set to 1 .

In quantitative thermography, it is commonly assumed to refer to the integral intensity of the target divided by that of the blackbody, by introducing the notion of effective emissivity $\varepsilon_{\Delta \lambda}$ : 


$$
L_{\Delta \lambda, e}(T)=\varepsilon_{\Delta \lambda} \times L_{\Delta \lambda, e}^{0}(T)
$$

This expression leads to the well-known one for the measured radiation intensity of an opaque target surface:

$$
L_{\Delta \lambda}\left(T_{\text {app. }}\right)=\varepsilon_{\Delta \lambda} L_{\Delta \lambda}^{0}(T)+\left(1-\varepsilon_{\Delta \lambda}\right) \times L_{\Delta \lambda}^{0}\left(T_{\text {env. }}\right)
$$

where $r_{\Delta \lambda}=\left(1-\varepsilon_{\Delta \lambda}\right)$ is the effective reflectivity of the target surface. The awareness of $\varepsilon_{\Delta \lambda}$ and of the equivalent thermal environment temperature enable the determination of the target surface intensity $L_{\Delta \lambda}^{0}(T)$ from the measured apparent one $L_{\Delta \lambda}\left(T_{\text {app. }}\right)$ and consequently permit the calculation of the emitted temperature using the calibration curve of the infrared system. According to the typical procedure of temperature measurement, any operator has to quantify and enter only these two values in the microcomputer system. Reflected temperature is defined as 'the temperature of the energy incident upon and the reflected one from the measurement surface of a specimen' and its determination is at present standardised [5]. Quantifying the effective emissivity $\varepsilon_{\Delta \lambda}$ remains a difficult problem. Its value depends not only on the emission properties of the target (spectral emissivity, state surface, thickness...), but also on the spectral characteristics of the IR system (bandwidth detection, spectral response of the detectors, optical transmittance, etc.) and on the view angle. As pointed out in [6], the classical formula which permits to calculate the effective emissivity as the mean value of spectral emissivity weighted by the blackbody spectral radiance should be used only in the case of emitters and IR systems, which exhibit non-selective spectral response. Employing the effective emissivity such as the spectral emissivity averaged within the spectral range of the IR system can bring significant errors and should generally be avoided. To meet this difficulty, a relevant approach is to determine in situ the effective emissivity using the IR system itself under experimental conditions.

\section{Effective emissivity characterisation}

From Equation (4), it is observed that the expression of the effective emissivity $\varepsilon_{\Delta \lambda}$ is trivial. Numerical values can be extracted with:

$$
\varepsilon_{\Delta \lambda}=\frac{L_{\Delta \lambda}\left(T_{\text {app. }}\right)-L_{\Delta \lambda}^{0}\left(T_{\text {env. }}\right)}{L_{\Delta \lambda}^{0}(T)-L_{\Delta \lambda}^{0}\left(T_{\text {env. }}\right)}
$$

Its implementation requires the knowledge of each involved temperature and of the calibration curve of IR thermometer. Supposing that $T$ is the target temperature of the surface, then the equivalent blackbody radiation intensity can be found in terms of numerical levels using the calibration curve of the camera. Alike, apparent temperature $T_{\text {app. }}$ gives equivalent measured radiation intensity, and the environment one $T_{\text {env. }}$ gives the equivalent radiation intensity of the environment. Effective emissivity $\varepsilon_{\Delta \lambda}$ is thereby calculated by using Equation (5). By varying the temperature of the target, it is also possible to map effective emissivity on large temperature ranges.

Despite its simplicity, Equation (5) enables stable and reproducible measurements and it has been recently implemented in [7]. Also, this integral form of normal effective emissivity is consistent with the spectral form used in [8] in which the authors have built up 
a portable FTIR spectrometer for in situ directional measurement of monochromatic emissivity. Another method of effective emissivity characterisation is described in [9]. Emissivity is measured in a situation of radiometric balance (surface emission + surface reflection $=$ environment emission) and it requires a strict control of all sources of thermal radiation of the scene. A small additional exposure of the target disrupts the balance so as to approximate the changes of radiance with linear variations. The emissivity can thus be calculated from radiometric temperature differences. With the aid of a thermostatic chamber surrounding the target, it is then possible to map the effective emissivity with temperatures.

\section{Experimental set-up}

As our aim is to know the hot plate temperature with accuracy before all deposition considerations, the hot plate is placed so as to determine the radiation intensities and temperatures in Equation (4). The experimental set-up done for these determinations is shown in Figure 2. The considered hot plate is made of aluminium nitride (AlN). Its area is $75 \times 25 \mathrm{~mm}^{2}$ and its thickness is $3 \mathrm{~mm}$. The plate is heated by embedded resistive wires. A type $\mathrm{K}$ thermocouple is also embedded in the plate. Both the electrical power and the temperature can be controlled by means of a Proportional Integral Derivative (PID) controller. The whole equipment, hot plate and regulation system, is marketed by the Watlow Company.

The hot plate is placed in a surrounding cardboard in order to have a homogeneous environment. Temperatures are evaluated by means of a VOx uncooled IRFPA camera placed vertically to the hot plate. The camera is a commercial one type FLIR E300, measuring in LWIR domain with $320 \times 240$ detectors. The calibration of the camera was obtained by means of software capabilities on two ranges $\left[-20^{\circ} \mathrm{C}, 120^{\circ} \mathrm{C}\right]$ and $\left[50{ }^{\circ} \mathrm{C}, 500^{\circ} \mathrm{C}\right]$. The radiation intensity mapping of the hot plate is indeed taken with the camera for set point temperatures given by the $\mathrm{K}$ type thermocouple in the range of $40^{\circ} \mathrm{C}$ up to $540^{\circ} \mathrm{C}$ in $20^{\circ} \mathrm{C}$ steps.

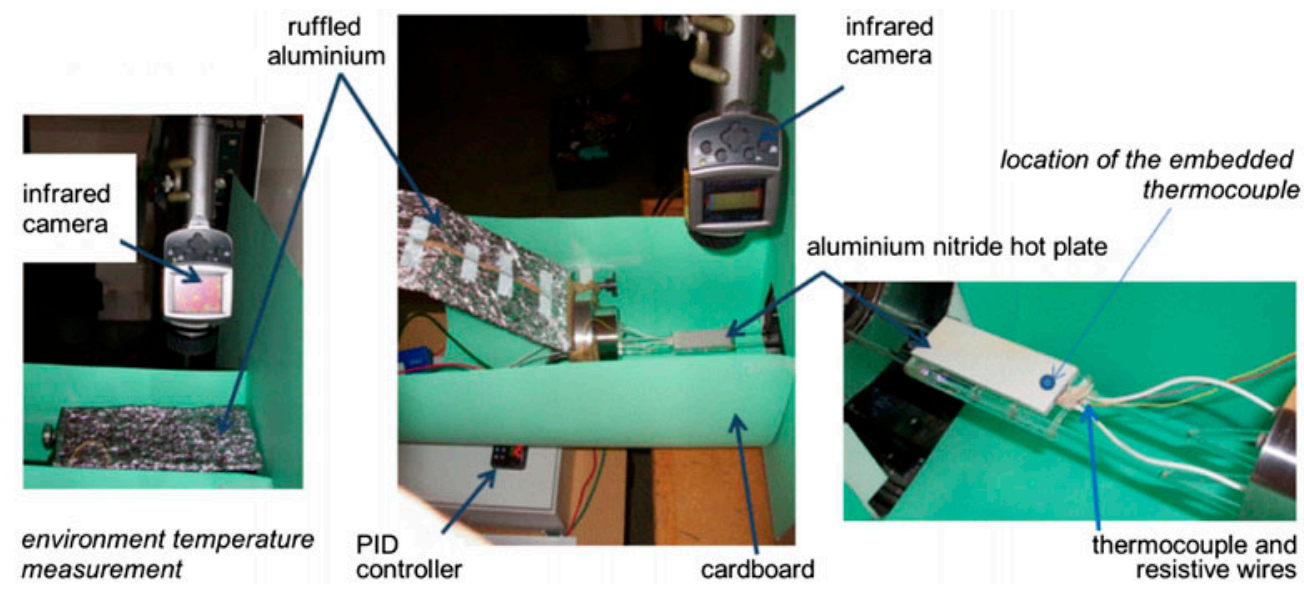

Figure 2. Experimental set-up. 


\section{Calibration curve of the camera}

Calibration refers to the sets of operations which establish the relationship between the spectral radiation absorbed by each detector of an IRFPA camera (camera level or thermosignal) and the corresponding known value of spectral blackbody radiation emitted by the source at a temperature $T$. An IRFPA camera is typically calibrated over a range of temperatures. Calibration is performed over a spectral bandwidth $\Delta \lambda$. The relationship between the camera levels (thermosignals) and the blackbody temperatures constitutes the calibration curve of the camera.

When we use commercial IR camera, we only dispose of thermosignals and apparent temperatures. We have to keep in mind that thermosignals are subject to numerous corrections and formatting to make them compatible with commercial software. Thermosignals are commonly quantified in object signal unit (OS). Thus, we must check first the connection between these formatted quantities and the radiance to test the metrological nature of the thermosignals in order to generalise the treatment of any IR camera.

Due to this, the first step consists in getting the thermosignal/apparent temperature correspondence. As shown in the general equation of thermography, the overall radiometric emission provides directly the apparent temperature after thermosignal/temperature transcription. In practice, this is achieved by specifying for the software an effective emissivity of 1 and an observation distance of zero (no atmospheric contribution). The associated thermosignals are then extracted on any thermogram whose apparent temperatures cover the temperature range of the IR camera.

(a)
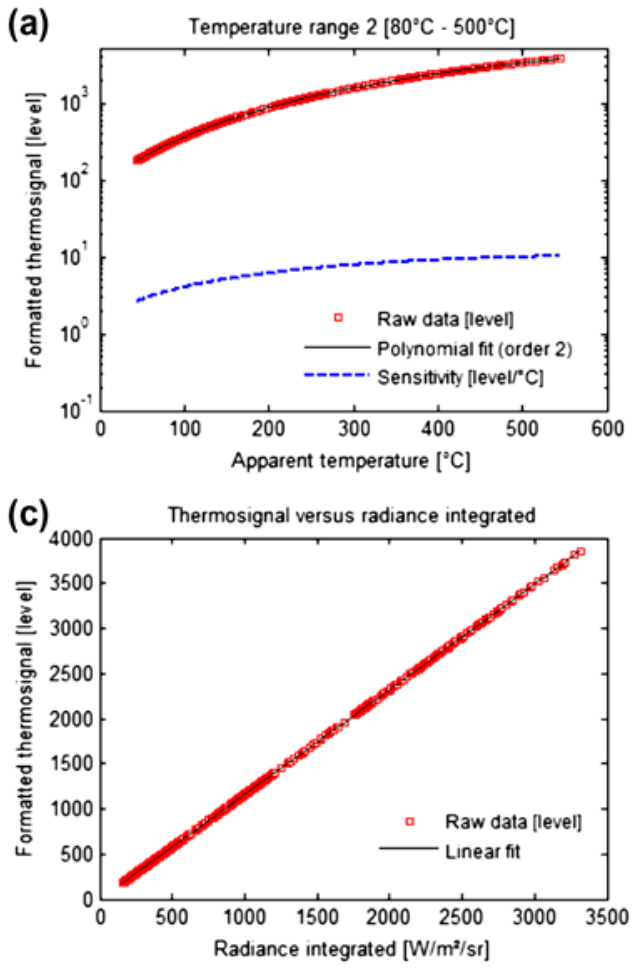
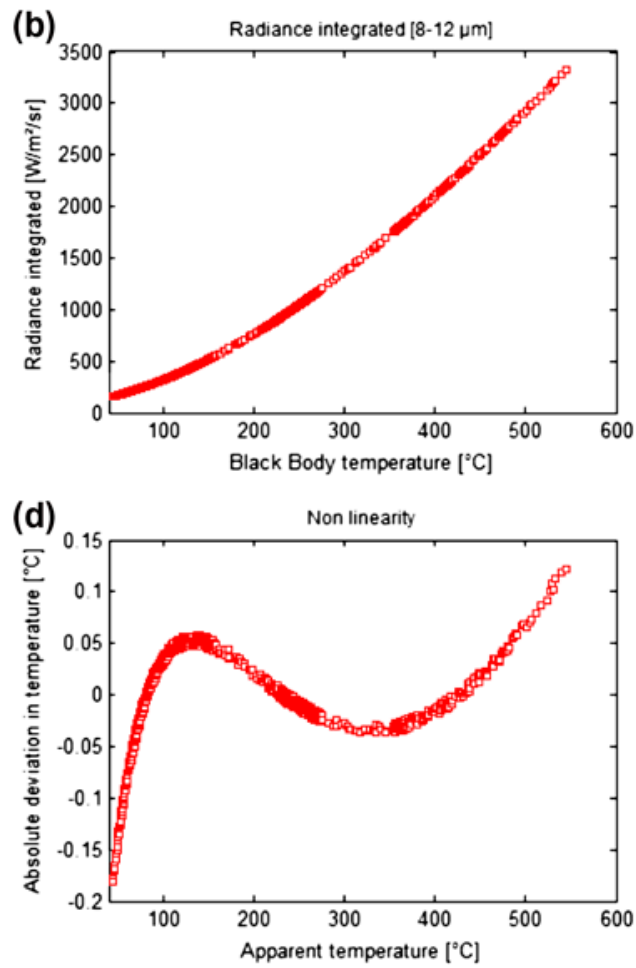

Figure 3. (a) Thermosignal/effective temperature correspondence with sensitivity; (b) Extension of the calculation of the spectral radiance integrated over temperature range of the camera; (c) Thermosignal/ integral radiance correspondence; (d) Deviation from linearity. 
Unfortunately, the problem of the position of the effective spectral band is difficult to solve since it depends on numerous choices made by the manufacturer which are not often explained. In the case of VOx uncooled camera, the spectral band is commonly defined in the range $7.5-14 \mu \mathrm{m}$. Once this characteristic is admitted, we can calculate the spectral radiance integrated over the window $\Delta \lambda$ for the two temperature ranges and then plot the curve thermosignals vs. integrated radiance. The two temperature ranges exhibit a linear characteristic (slope of roughly $0.78 \mathrm{OS} /\left[\mathrm{W} \mathrm{m}^{-2} \mathrm{sr}^{-1}\right]$, value at the origin of less than seven signal units), which means that raw thermosignals are effectively normalised to the radiance of blackbody, as it is usually the case when we record the thermogram to allow post-processing computer. In terms of temperature, the residual deviation is less than $0.25^{\circ} \mathrm{C}$ on the temperature range of the camera. By testing the sensitivity of linearity to spectral band, we observe a cancellation of the value at the origin while we reduce the spectral bandwidth. For the band $[8,12.5 \mu \mathrm{m}]$, the temperature range 1 is strictly linear (slope $=1.0$ ) with integrated radiance (value at origin roughly $0.2 \mathrm{OS}$ which represents a temperature offset less than $0.1^{\circ} \mathrm{C}$ ). For temperature range 2, the same behaviour is fully met for the band $[8,12 \mu \mathrm{m}]$ (value at the origin: $1 \mathrm{OS},<0.3^{\circ} \mathrm{C}$ ). Illustrations are displayed in Figure 3. These last bands can be best suited to formatted data during the recording of the thermogram and to adapt the decompression process to all cameras of the same family. We checked this assumption for ThermaCAM E 4 $(160 \times 120$ VOx uncooled IRFPA $)$ and ThermaCAM PM $280(320 \times 240$ VOx uncooled IRFPA) whose characteristic thermosignal - integrated radiance is the same as for the camera E 300 utilised here. E 300 camera calibration curve is then determined by fitting the thermosignal/apparent temperature characteristic using a two-degree polynomial function.

\section{Effective emissivity calculation}

The thermocouple which equips the aluminium nitride hot plate gives bulk and then surface temperature close to it. Its location is indicated on the right part of Figure 2. The supplied power via the PID controller allows us to stabilise the temperature from 40 to $540{ }^{\circ} \mathrm{C}$ with $20^{\circ} \mathrm{C}$ steps. For each stabilised temperature, we recorded apparent, surface and environment

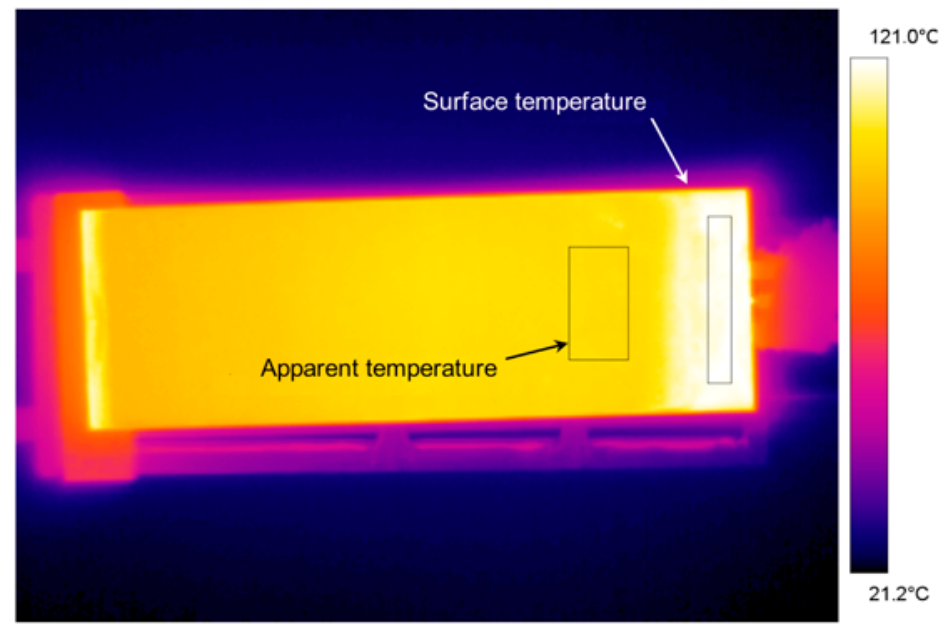

Figure 4. Analysis areas giving apparent and exact surface temperatures on the aluminium nitride plate. The two strips at the ends of the plate appear to be high emissivity emitters and serve as temperature reference. 


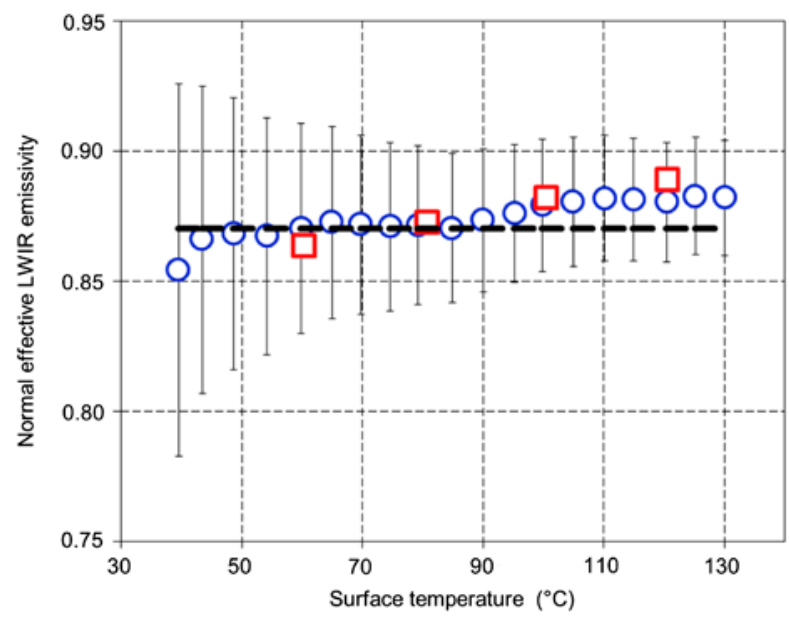

Figure 5. Normal effective LWIR emissivity of sintered AlN plate. Round marks: camera range $\left[-20^{\circ}\right.$ $\left.\mathrm{C}, 120^{\circ} \mathrm{C}\right]$; square marks: camera range $\left[80^{\circ} \mathrm{C}, 500^{\circ} \mathrm{C}\right]$.Dashed line: average emissivity obtained from classical ellipsometry method on AlN amorphous thick film.

average temperatures within the areas shown in Figure 4. In our case, the hot plate surface material happens not to be the same on the surface of the whole plate. There are three different material surfaces which can be noticed in Figure 5. To our good luck, the material surface above the thermocouple appears to be an excellent emitter. The surface temperature is then recorded on this area. Its effective emissivity neighbours 1 since insignificant error is noted when we compare thermocouple and apparent temperatures. This surface material is probably an aluminium oxide thin film well-known for having high spectral emissivity over LWIR bandwidth [10]. This aluminium oxide film was certainly formed during the sintering process. The same material is present on the other end of the plate.

The environment temperature is recorded by means of a rigid ruffled aluminium foil according to Standard Test Methods which covers entirely the plate (see the left picture of Figure 2).

Since we dispose of each temperature, it is possible to calculate IR camera levels associated by using the calibration curves and finally to compute spectral emissivity given by Equation (5). For clarity, we remove $\Delta \lambda$ index since it is now implicit that it concerns LWIR standard band. Uncertainty on normal effective LWIR emissivity can be evaluated by using Equation (6) (see Appendix 1):

$$
\frac{\Delta \varepsilon_{\Delta \lambda}}{\varepsilon_{\Delta \lambda}}=\frac{\Delta L\left(T_{\text {app. }}\right)+\Delta L^{0}\left(T_{\text {env. }}\right)}{\left|L\left(T_{\text {app. }}\right)-L^{0}\left(T_{\text {env. }}\right)\right|}+\frac{\Delta L^{0}(T)+\Delta L^{0}\left(T_{\text {env. }}\right)}{\left|L^{0}(T)-L^{0}\left(T_{\text {env. }}\right)\right|}
$$

Levels are determined with an absolute precision due to absolute uncertainty on radiometric temperature. Level uncertainties are deduced from $\Delta L(T)=s \times \Delta T$ where $s$ is the sensitivity in $\mathrm{OS} /{ }^{\circ} \mathrm{C}$. Radiometric temperature uncertainties are previously evaluated using a blackbody calibrator on the range $\left[40^{\circ} \mathrm{C}, 300^{\circ} \mathrm{C}\right]$ and show an uncertainty of $\pm 0.5 \%$ in Celsius degree on the temperature range $1\left[-20^{\circ} \mathrm{C}, 120^{\circ} \mathrm{C}\right]$ and of $\pm 0.7 \%$ for the other camera range $2\left[80^{\circ} \mathrm{C}, 500^{\circ} \mathrm{C}\right]$. Environment temperature is evaluated within arbitrary $\pm 0.5^{\circ} \mathrm{C}$. The obtained normal effective LWIR emissivity results are provided in Figure 5 for surface temperatures going from 40 up to $120^{\circ} \mathrm{C}$ for both the camera ranges. Taking into 


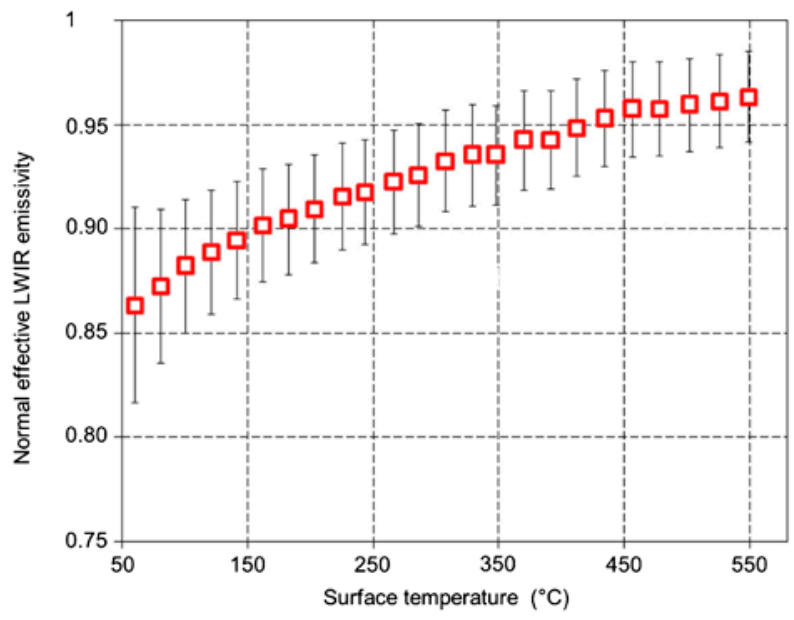

\begin{tabular}{|cccccc|}
\hline $\begin{array}{c}\text { Surface } \\
\text { temp. } \\
{\left[{ }^{\circ} \mathrm{C}\right]}\end{array}$ & $\begin{array}{c}\text { Normal effective } \\
\text { LWIR emissivity }\end{array}$ & $\begin{array}{c}\text { absolute } \\
\text { uncertainty } \\
{[ \pm]}\end{array}$ & $\begin{array}{c}\text { Surface } \\
\text { temp. } \\
{\left[{ }^{\circ} \mathrm{C}\right]}\end{array}$ & $\begin{array}{c}\text { Normal effective } \\
\text { LWIR emissivity }\end{array}$ & $\begin{array}{c}\text { absolute } \\
\text { uncertainty } \\
{[ \pm]}\end{array}$ \\
\hline 60,3 & 0,86 & 0,05 & 307,7 & 0,93 & 0,02 \\
\hline 80,8 & 0,87 & 0,04 & 329 & 0,94 & 0,02 \\
\hline 100,5 & 0,88 & 0,03 & 348 & 0,94 & 0,02 \\
\hline 120,6 & 0,89 & 0,03 & 370,3 & 0,94 & 0,02 \\
\hline 141,8 & 0,89 & 0,03 & 391,6 & 0,94 & 0,02 \\
\hline 161,9 & 0,9 & 0,03 & 411,7 & 0,95 & 0,02 \\
\hline 182,5 & 0,9 & 0,03 & 433,9 & 0,95 & 0,02 \\
\hline 203,1 & 0,91 & 0,03 & 456,6 & 0,96 & 0,02 \\
\hline 225,4 & 0,92 & 0,03 & 477,6 & 0,96 & 0,02 \\
\hline 243,4 & 0,92 & 0,03 & 501,8 & 0,96 & 0,02 \\
\hline 266,8 & 0,92 & 0,02 & 525,8 & 0,96 & 0,02 \\
\hline 286,4 & 0,93 & 0,02 & 548,6 & 0,96 & 0,02 \\
\hline
\end{tabular}

Figure 6. Temperature dependence of normal effective LWIR emissivity of AlN plate with the corresponding found data.

account uncertainty, effective emissivity is connected within the two ranges of determination. The normal effective LWIR emissivity value of aluminium nitride had been evaluated in another way by a classical method of ellipsometry which allowed to find complex refractive indexes [11]. Considering the evaluated optical characteristics of aluminium nitride amorphous thick films, aluminium nitride emissivity is about 0.87 . Thus, present effective emissivity measurements are in good agreement with this standard method of calculation of average spectral emissivity $[12,13]$.

By extending the emissivity determination to the second temperature range $\left[80^{\circ} \mathrm{C}\right.$, $500{ }^{\circ} \mathrm{C}$ ], the effective emissivity is found temperature dependent as represented in Figure 6. In spite of uncertainty, temperature dependence points out a deterministic behaviour which will be interesting to correlate to spectral emissivity within the effective spectral band and to the effect of surface porosity [14].

\section{Temperature profiles}

Since the normal effective LWIR emissivity is known, the true hot plate temperature can be deduced. The temperatures are first analysed on several segments taken on the hot plate 


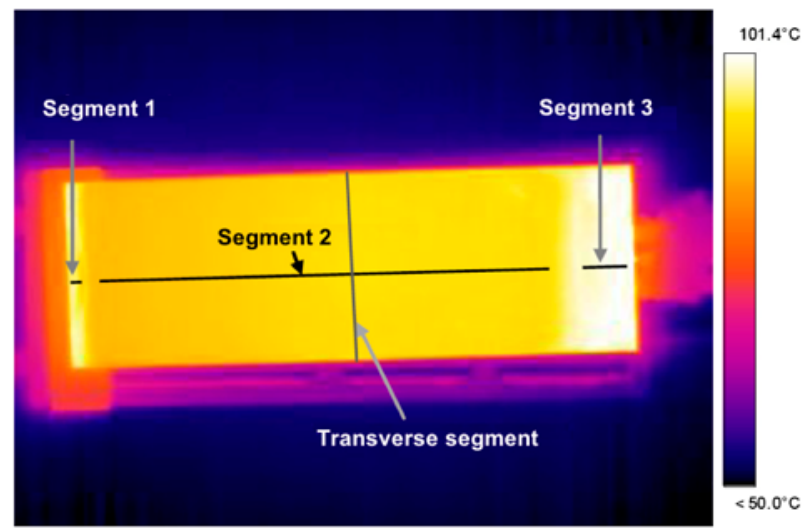

Figure 7. Analysed segments: 1, 2 and 3 in the longitudinal direction and the one in the transverse direction.

longitudinal midline as indicated in Figure 7. The apparent temperatures from segment 2 are corrected using normal emissivity and the environment temperature follows Equation (5). "Segments" 1 and 3 are supposed to give directly the exact surface temperature since the surface material is a quasi-perfect emitter. A good agreement between the segment extremities is then expected. Abscissa is normalised to $75 \mathrm{~mm}$ corresponding to the plate length. Temperature values are secondly observed on the transverse midline of the plate and normalised to $25 \mathrm{~mm}$ corresponding to the plate width.

Uncertainty for the surface temperature is evaluated using $\Delta T=\Delta L^{0}(T) / s$ where $\Delta L^{0}(T)$ is the uncertainty on the blackbody emitter level $L^{0}(T)$ given by Equation (7) (see Appendix 2):

$$
\frac{\Delta L^{0}(T)}{L^{0}(T)}=\frac{\Delta L\left(T_{\text {app. }}\right)}{\varepsilon_{\Delta \lambda} L^{0}(T)}+\left|1-\frac{L^{0}\left(T_{\text {env. }}\right)}{L^{0}(T)}\right| \times \frac{\Delta \varepsilon_{\Delta \lambda}}{\varepsilon_{\Delta \lambda}}+\left|1-\frac{L\left(T_{\text {app. }}\right)}{\varepsilon_{\Delta \lambda} L^{0}(T)}\right| \times \frac{\Delta L^{0}\left(T_{\text {env. }}\right)}{L^{0}\left(T_{\text {env. }}\right)}
$$
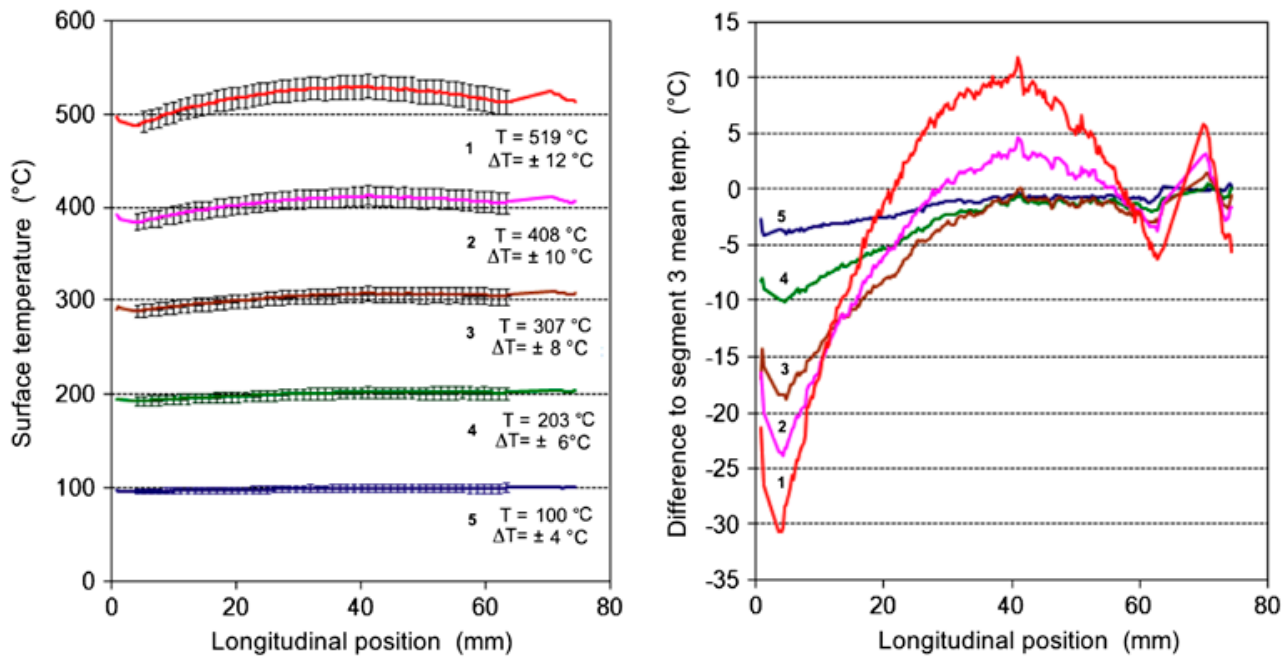

Figure 8. Left: lengthwise temperature profiles with the uncertainty bars. Right: temperature difference compared to "segment 3" mean temperature. 

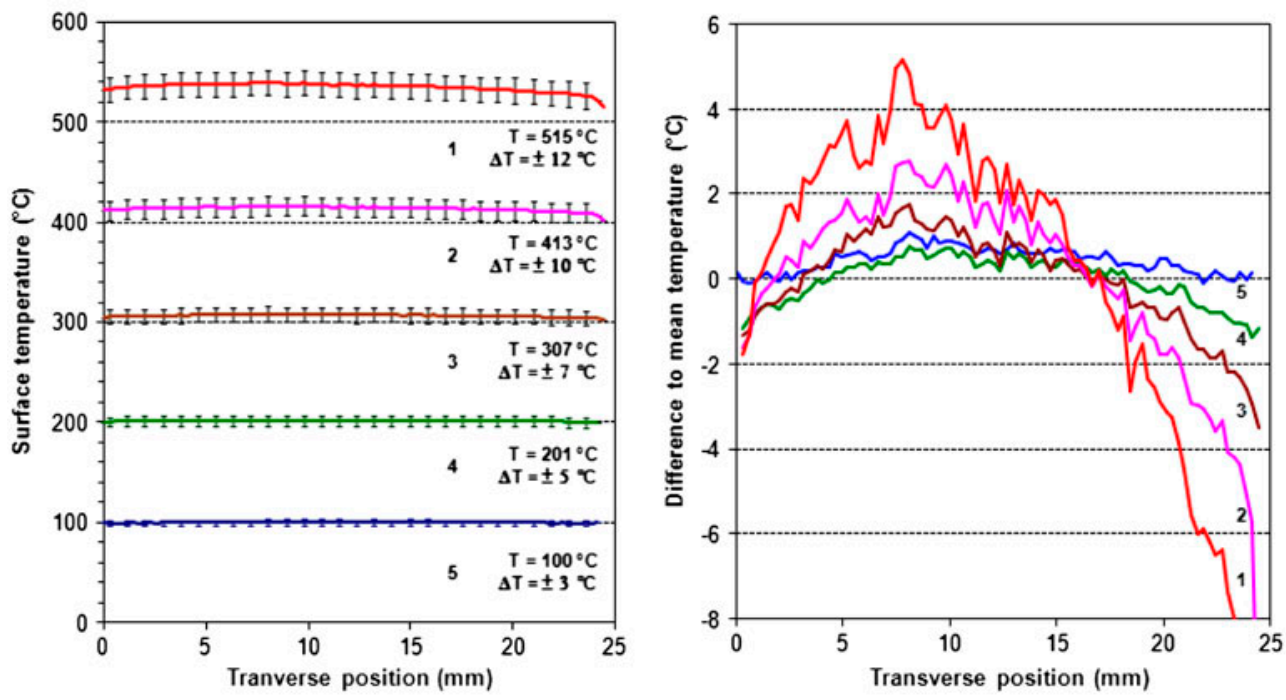

Figure 9. Left: transverse temperature profiles with the uncertainty bars. Right: difference with regard to mean temperature.

The absolute temperature uncertainties are unchanged as in the emissivity calculation (see Section 6). The temperature results are shown in Figure 8 for the longitudinal direction and in Figure 9 for the transversal one for several set point temperatures going from 100 up to $500{ }^{\circ} \mathrm{C}$. The calculated uncertainty bars with Equation (7) are indicated. The temperature differences compared to the mean value are displayed on the right part of Figures 8 and 9.

A good agreement is verified at the segment extremities for the lengthwise temperature profiles. The temperature uncertainty increases when the temperature rises mainly because of the emissivity uncertainty. However, in percentage, it decreases with temperature. The uncertainty is found in the order of $4 \%$ for the considered lower temperatures and up to $2.5 \%$ for the upper ones. In our case $\left(\varepsilon_{\Delta \lambda} \approx 1\right)$, uncertainties are mainly restricted to the ones of emissivity and by exactitude of IR camera (see Appendix 2).

The temperature differences augment when the hot plate overall temperature is more important. The difference in the longitudinal direction is bigger than the one in the transverse direction because the length is equal to three times the width of the hot plate. Nevertheless, in terms of percentage in Celsius degree, the temperature difference is inferior to $8 \%$ of the mean temperature value for the lengthwise midline and inferior to $2.5 \%$ for the transverse one. So, a good homogeneity is found for the hot plate temperature.

\section{Conclusion}

The temperature of an aluminium nitride hot plate which can be used for spray CVD has been determined by a thermographic method. First, the calibration curve of the used IR camera was obtained by software capacities. Basic spectral analysis shows the consistency of the calibration curve thus obtained. The normal effective emissivity of the aluminium nitride of the hot plate was then characterised by measuring apparent, surface and environment temperatures for both the camera standard temperature ranges $\left[-20^{\circ} \mathrm{C}, 120^{\circ} \mathrm{C}\right]$ and $\left[50^{\circ} \mathrm{C}, 550^{\circ} \mathrm{C}\right]$. Measured values on these two ranges are convergent. The cartography of effective emissivity 
for temperatures ranging from 30 to $550{ }^{\circ} \mathrm{C}$ was done with a precision better than $3 \%$. It shows strong deterministic temperature dependence. Knowing the effective emissivity, the true temperature profiles were deduced for both the mid longitudinal and transverse directions of the hot plate. The temperature uncertainty in Celsius degree is as well calculated with an accuracy ranging from $4 \%$ for low temperatures to $2 \%$ for higher ones. Indeed, the uncertainty lessens in percentage when the temperature increases, which makes it worthwhile employing the present thermographic approach for high temperature evaluation. Finally, good temperature homogeneity is verified with non-homogeneity inferior to $8 \%$ of the mean temperature. So, our next step is thin film deposition with a glass substrate laid on the heater plate. Numerical simulation is also another interesting perspective since the aluminium nitride emissivity values are known with the temperature.

\section{Acknowledgements}

Both the research teams express their full gratitude to Mrs Wilhelmina Logerais, a native English speaker, for lending a hand to the English wording of this paper.

\section{References}

[1] Lavernia EJ, Wu Y. Spray atomization and deposition. New York (NY): Wiley; 1996.

[2] Zhou ZB, Cui RQ, Pang QJ, Wang YD, Meng FY, Sun TT, Ding ZM, Yu XB. Preparation of indium tin oxide films and doped tin oxide films by an ultrasonic spray CVD process. Applied Surface Science. 2001 Mar 15;172(3-4):245-52.

[3] De La L, Olvera M, Gómez H, Maldonado A. Doping vacuum annealing, and thickness effect on the physical properties of zinc oxide films deposited by spray pyrolysis. Solar Energy Materials and Solar Cells. 2007 Sep 22;91(15-16):1449-53.

[4] Girtan M, Logerais PO, Bouteville A. Substrate thermal profiles in spray-CVD reactor. Journal of Optoelectronics and Advances Materials. 2006 Feb;8(1):144-7.

[5] ASTM E1862-97(2002)-e1. Standard test methods for measuring and compensating for reflected temperature using infrared imaging radiometers. 2002.

[6] Chrzanowski K. Problem of determination of effective emissivity of some materials in MIR range. Infrared Physics and Technology. 1995;36(3):679-84.

[7] Marinetti S, Cesaratto PG. Emissivity estimation for accurate quantitative thermography. NDT and E International. 2012;51:127-34.

[8] Korb AR, Dybwad P, Wadsworth W, Salisbury JW. Portable FTIR spectrometer for field measurements of radiance and emissivity. Applied Optics. 1996;35:1679-92.

[9] Vainer BG. Focal plane array based infrared thermography in fine physical experiment. Journal of Physics D: Applied Physics. 2008;41:065102 (12 pp).

[10] White FM. Heat transfer. Reading (MA): Addison-Wesley; 1984.

[11] Palik ED. Handbook of optical constants of solids. New York (NY): Academic Press; 1998.

[12] Ivanov SN, Popov PA, Egorov GV, Sidorov AA, Kornev BI, Zhukova LM, Ryabov VP. Thermophysical properties of aluminium nitride ceramic. Physics of the Solis State. 1997 Jan 1;39(1):81-3.

[13] Sciti D, Winterhalter F, Bellosi A. Oxidation behaviour of a pressureless sintered AlN-SiC composite. Journal of Material Science. 2004 Jan 1;39:6965-73.

[14] Rozenbaum O, De Sousa Meneses D, Echegut P. Texture and porosity effects on the thermal radiative behavior of alumina ceramics. International Journal of Thermophysics. 2009 Apr 1;30 (2):580-90. 


\section{Appendix 1. Uncertainty on normal effective LWIR emissivity}

Uncertainty can be calculated using logarithm or partial derivative methods. Equation (5) gives the expression of effective emissivity $\varepsilon_{\Delta \lambda}$. By taking the logarithm, we find:

$$
\ln \left(\varepsilon_{\Delta \lambda}\right)=\ln \left(L\left(T_{\text {app }}\right)-L^{0}\left(T_{\text {env. }}\right)\right)-\ln \left(L^{0}(T)-L^{0}\left(T_{\text {env. }}\right)\right)
$$

We apply the derivative of the expression above:

$$
\frac{d \varepsilon_{\Delta \lambda}}{\varepsilon_{\Delta \lambda}}=\frac{d L\left(T_{\text {app }}\right)+d L^{0}\left(T_{\text {env. }}\right)}{L\left(T_{\text {app }}\right)-L^{0}\left(T_{\text {env. }}\right)}+\frac{d L^{0}(T)+d L^{0}\left(T_{\text {env. }}\right)}{L^{0}(T)-L^{0}\left(T_{\text {env. }}\right)}
$$

Finally, we maximise the different variations by taking the absolute value of each term:

$$
\frac{\Delta \varepsilon_{\Delta \lambda}}{\varepsilon_{\Delta \lambda}}=\frac{\Delta L\left(T_{\text {app }}\right)+\Delta L^{0}\left(T_{\text {env. }}\right)}{\left|L\left(T_{\text {app }}\right)-L^{0}\left(T_{\text {env. }}\right)\right|}+\frac{\Delta L^{0}(T)+\Delta L^{0}\left(T_{\text {env. }}\right)}{\left|L^{0}(T)-L^{0}\left(T_{\text {env. }}\right)\right|}
$$

\section{Appendix 2. Uncertainty on surface temperature}

Equation (4) gives the expression of the radiance of the target in the classical model. We rearrange the relation so as to extract the radiance of blackbody referred to the surface temperature of the target:

$$
L^{0}(T)=\frac{L\left(T_{\text {app }}\right)-L^{0}\left(T_{\text {env. }}\right)}{\varepsilon_{\Delta \lambda}}+L^{0}\left(T_{\text {env. }}\right)
$$

Using the method of partial derivative, we find:

$$
d L^{0}(T)=\frac{d L\left(T_{\text {app }}\right)}{\varepsilon_{\Delta \lambda}}-\left[\frac{L\left(T_{\text {app }}\right)-L^{0}\left(T_{\text {env. }}\right)}{\varepsilon_{\Delta \lambda}}\right] \times \frac{d \varepsilon_{\Delta \lambda}}{\varepsilon_{\Delta \lambda}}+\left[\frac{\varepsilon_{\Delta \lambda}-1}{\varepsilon_{\Delta \lambda}}\right] \times d L^{0}\left(T_{\text {env. }}\right)
$$

From Equation (5), we have $L\left(T_{\text {app }}\right)-L^{0}\left(T_{\text {env. }}\right)=\varepsilon_{\Delta \lambda} \times\left(L^{0}(T)-L^{0}\left(T_{\text {env. }}\right)\right)$ and $\varepsilon_{\Delta \lambda}-1=$ $\frac{\varepsilon_{\Delta \lambda} \times L^{0}(T)-L\left(T_{\text {app }}\right)}{L^{0}\left(T_{\text {env. }}\right)}$.

Thus:

$$
d L^{0}(T)=\frac{d L\left(T_{\text {app }}\right)}{\varepsilon_{\Delta \lambda}}-\left[L^{0}(T)-L^{0}\left(T_{\text {env. }}\right)\right] \times \frac{d \varepsilon_{\Delta \lambda}}{\varepsilon_{\Delta \lambda}}-\left[L^{0}\left(T_{\text {env. }}\right)-\frac{L\left(T_{\text {app }}\right)}{\varepsilon_{\Delta \lambda}}\right] \times \frac{d L^{0}\left(T_{\text {env. }}\right)}{L^{0}\left(T_{\text {env. }}\right)}
$$

Finally, we divide the above equation by $L^{0}(T)$ and then we maximise the different variations by taking the absolute value for each term:

$$
\frac{\Delta L^{0}(T)}{L^{0}(T)}=\frac{\Delta L\left(T_{\text {app }}\right)}{\varepsilon_{\Delta \lambda} \times \Delta L^{0}(T)}+\left|1-\frac{L^{0}\left(T_{\text {env. }}\right)}{L^{0}(T)}\right| \times \frac{\Delta \varepsilon_{\Delta \lambda}}{\varepsilon_{\Delta \lambda}}+\left|1-\frac{L\left(T_{\text {app }}\right)}{\varepsilon_{\Delta \lambda} \times L^{0}(T)}\right| \times \frac{\Delta L^{0}\left(T_{\text {env. }}\right)}{L^{0}\left(T_{\text {env. }}\right)}
$$


It is interesting to study the case of $T>>T_{\text {env. }}$ and $e_{\Delta \lambda} \approx 1$ for which uncertainty can be approximated by:

$$
\frac{\Delta L^{0}(T)}{L^{0}(T)} \approx \frac{\Delta L\left(T_{\text {app }}\right)}{L\left(T_{\text {app }}\right)}+\frac{\Delta \varepsilon_{\Delta \lambda}}{\varepsilon_{\Delta \lambda}}
$$

This relation shows that uncertainties in temperature are mainly restricted to the ones related to the effective emissivity $\varepsilon_{\Delta \lambda}$ and to the exactitude of the IR camera. 